\title{
La capacidad de tolerancia al ejercicio predice las complicaciones postquirúrgicas graves
}

Self-reported Exercise Tolerance and the risk of serious perioperative complications. Arch Intern Med 1999; 159: 2185-2192

\section{Objetivo}

Determinar la relación entre la tolerancia autorreportada al ejercicio y la incidencia de complicaciones postquirúrgicas graves en cirugía mayor no cardíaca.

\section{Diseño}

Estudio prospectivo de cohorte con una mediana de seguimiento de 20 meses.

\section{Lugar}

Consultorio de evaluación prequirúrgica y sala internación de un hospital universitario de tercer nivel (Washington Medical Center-Seattle USA).

\section{Participantes}

De 951 pacientes referidos consecutivamente para evaluación prequirúrgica durante el período octubre 1995 a junio 1997, el 94\% (896) consintieron en participar. De estos el $26 \%$ (229) fueron excluidos por ser sometidos a cirugía menor. A 67 pacientes de los 667 restantes se les canceló la intervención ingresando en definitiva 600 pacientes.

\section{Evaluación de la capacidad funcional y otros factores de Riesgo}

Se evaluó por medio de interrogatorio y exámen físico los riesgos previos y se realizaron los estudios complementarios normatizados para este tipo de evaluación. Se relevó en cada paciente la capacidad para caminar de 0 a 4 cuadras y de subir hasta 4 pisos por escaleras sin experimentar limitación física.

Se utilizaron puntajes (scores) previamente validados como los de Goldman y Destky para la evaluación de riesgo cardiovascular y el ASA para el riesgo general.

\section{Resultados Principales}

Tolerancia al ejercicio y eventos graves:

Los pacientes con mala tolerancia reportada sufrieron más complicaciones perioperatorias $(20.4 \%$ vs $10.45 \%, p<0.001)$. Ellos tuvieron específicamente más eventos coronarios $(p<0.02)$, cardiovasculares $(p<0.04)$ y neurológicos ( $p<0.03)$ que los que poseían buena tolerancia. La probabilidad de eventos graves fue inversamente proporcional al número de cuadras que podían caminar $(\mathrm{p}=0.006)$ o pisos que podían subir $(\mathrm{p}=0.01)$

\begin{tabular}{lccc}
\hline $\begin{array}{l}\text { Eventos } \\
\text { Periopeatorios }\end{array}$ & $\begin{array}{l}\text { Buena tolerancia al } \\
\text { ejercicio }(\mathbf{n}=269)\end{array}$ & $\begin{array}{l}\text { Mala tolerancia al } \\
\text { al ejercicio }(\mathbf{n = 3 4 3 )}\end{array}$ & $\mathbf{P}$ \\
\hline Cardiovasculares & $14(5.2)$ & $33(9.6)$ & 0.04 \\
\hline Pulmonares & $17(6.3)$ & $31(9.0)$ & 0.21 \\
\hline Neurológicos & $6(2.2)$ & $20(5.8)$ & 0.03 \\
\hline $\begin{array}{l}\text { Pase a UTI } \\
\text { no esperado }\end{array}$ & $15(5.6)$ & $38(11.1)$ & 0.02 \\
\hline
\end{tabular}

Otros factores de riesgo y eventos graves:

a) Del paciente

Luego de realizar un análisis a través del método de regresión logística, además de la mala la tolerancia al ejercicio fueron predictores independientes la ICC, la demencia, la enfermedad de parkinson, y fumar más de 20 pack / years.

La edad no fue una variable predictora si bien los que tuvieron más complicaciones eran más viejos $(65.6+10.4$ vs $62.5+-15.4$, p-0.01).

b) Del tipo de cirugía

Los predictores fueron la duración de la anestesia ( $>0<8$ hs) y los procedimiento quirúrgicos torácicos y oncológicos

\section{Conclusiones}

La capacidad al ejercicio auto reportada fue predictor de complicaciones postquirúrgicas graves y fue de sencilla y rapida implementación en el consultorio.

\section{COMENTARIO}

Los cardiólogos fueron los primeros en observar la relación entre la tolerancia al ejercicio y la severidad de una enfermedad. (clasificaciones de la NYHA o Canadiense) ${ }^{1}$ y apartir de allí existen múltiples estudios que muestran la relación entre capacidad de ejercicio y morbimortalidad 2-34. No obstante este estudio impresiona ser el primero en la bibliografía que empleando un número grande de pacientes y una adecuada metodología demuestra que la tolerancia al ejercicio autorreportada de una manera sencilla predice complicaciones postquirúgicas graves en cirugía mayor no cardíaca.

En la evaluación prequirúrgica dos tipos de riesgos se deben considerar: los propios del paciente (comorbilidad cardiopulmonar principalmente) y los inherentes al tipo de cirugía: estrés fisiológico (anestesia, pérdida de sangre, dolor, hipotermia) y la complejidad del procedimiento ( número de planos atravesados, instrumentación, etc. ${ }^{5}$. En resumen la cirugía es fundamentalmente un evento de estrés psico-físico al que sometemos al paciente. Si poseen una mala tolerancia al ejercicio es de esperar que tampoco toleren bien el procedimiento Esta tolerancia alterada reflejaría no solo la comorbilidad sino también la capacidad funcional que con ella deviene.

El predictor individual más importante de mortalidad postquirúrgica es la presencia de enfermedad coronaria.

Se utilizaron "scores" previamente validados como los de Goldman y Destky para la evaluación del riesgo cardiovascular. Es sabido que estos no

predicen complicaciones en ciertos grupos de alto riesgo como por ejemplo los ancianos con baja capacidad funcional. En otras palabras estos pacientes pueden tener scores normales justamente por su actividad limitada en su vida diaria, en que no llegan a un grado de estrés como para disparar un evento cardiovascular. En estos pacientes, generalmente mayores de 70 años, si se sometieren a cirugía mayor están indicadas pruebas evocadoras de isquemia que sean independientes de su capacidad funcional tales como el talio-dipiridamol o el ecocardiograma con dobutamina ${ }^{6-7}$. De hecho el grupo de mala tolerancia en este artículo eran más añosos ( $61.2+-15.1$ vs 64.4+- $14.4 \mathrm{p}$ 0.008). El artículo no menciona si a los pacientes con mala tolerancia se les realizó alguna prueba de stress por ser considerados de riesgo para cirugía mayor. A pesar de ello, un dato interesante es que nuevamente la edad "per se" no fue predictor de complicaciones como previamente se ha reportado 8 -9.

La capacidad de ejercicio autorreportada parecería ser una medida de fácil implementación en un cuestionario de evaluación y una manera de incorporar una medida de actividad fisica objetiva más sencilla que la clasificación por METS y complementaria de los ABVD y AVDI que se utilizan para ancianos.

*Ver glosario

\section{Dr. Manuel Montero-0dasso}

Referencias

Servicio de Clínica Médica. Coordinador Médico del Centro de Prequirúrgico. Hospital Italiano de Buenos Aires.

1. Criteria Committe, New York Heart Association, Inc Disease of the herat and blood vessels. Nomenclature and Criteria for Diagnosis, 6th De. Boston, Little, Brown and Co. 1964

2. Sherman SE, D'agostina RB, CobbJL, Kannel WB. Does exercise reduce mortality rates in the elderly? Experience from the Framingham Heart Study. Am Heart J 1994;128:965-72

3. Shaper AG, Wannamethe G, Weatheral R. Physical activity and ischeamic heart disease in middle-aged men. Br Heart J 1991; 66:384-394

4. Hakim AA, Petrovich H, Burchfield CM, y col. Effects of walking on mortality among non-smoking retired men. N EngJ Med 1998; 338:94-99.

5. Eagle KA, Brundage BH, Chaitman Bret al. Guidelines for perioperative cardiovascular evaluations for non cardiac surgery: report of the American College of Cardiology/American Heart Association Task Force

6. J Am Coll Cardiol 1996:27:910-948

Thomas DR, Ritchie CS. Preoperative Assesment of Older Adults. J Am Geriatr Soc. 1995; 43:811-21

8. Montero-Odasso, Manuel. Evalaución perioperatoria en Ancianos Nexo Rev del Hospital Italiano. 1999; 5: 467-474

9. Dunlop WE, Rosenblood L, Lawrason L y col. Effects of age and severity of illness on outcome and length stay in geriatric surgical patients. Am J Surg 1993: 165:577-80 10. HHB jags 